\title{
LES COLÉOPTĖRES NUISIBLES AUX ANIMAUX DOMESTIQUES
}

\section{Par Jean THÉODORIDÉS}

Dans un travail précédent (Théodoridès, 1948), nous avions considéré les cas dans lesquels des coléoptères peuvent se rencontrer chez l'homme, s'y comportant comme des endoparasites accidentels. Nous voudrions, cette fois, mettre au point la question du danger relatif que présentent certains coléoptères pour les animaux domestiques, en Europe et aux colonies (1).

Les cas de parasitisme accidentel de coléoptères chez les animaux domestiques étant très rares, nous considérerons également, ici, ceux de ces insectes créant des lésions externes ou présentant une toxicité par ingestion.

Pour faciliter l'exposé, nous grouperons les cas connus par vertébré considéré.

\section{Mammifères}

\section{1. - Gheval.}

a. - Endoparasite accidentel. - Roberts (1931) rapporte le cas d'infestation de l'estomac d'un cheval, au Queensland, par Onthophagus granulatus Boh. (Scarabæidæ). L'animal en question était malade depuis trois semaines, se nourrissant très peu et présentant des troubles intestinaux. Après sa mort, un examen révéla la présence de nombreux coléoptères de l'espèce ci-dessus qui avaient perforé l'estomac. Roberts suppose que l'infestation est contractée soit par l'ingestion d'œufs ou de larves de l'insecte, soit par la pénétration d'imagos dans l'anus du cheval, alors que celui-ci est couché.

Cette dernière explication semble la plus vraisemblable et serait à rapprocher de celle donnée par différents auteurs, en ce qui concerne les cas de scarabiasis humains (cf. Théodoridès, 1948).

(1) Nous n'envisagerons pas ici les cas où des animaux domestiques (lapin, cobaye) servent de sujets d'expériences, au laboratoire, pour confirmer les propriétés de certains coléoptères vésicants (Pæderus, par exemple).

Ann. de Parasttologie, T. XXIV, $\mathrm{N}^{o s} 1-2 .-1949$, p. 116-123. 
b. - Lésions externes. - Des irritations locales (naseaux et bouche) chez les chevaux, causées par les sécrétions de Blaps mortisaga L. (Tenebrionidx), ont été signalées depuis déjà fort longtemps.

Tisserant (1886) décrit des chevaux présentant un soulèvement général de l'épiderme, se détachant en pelant, sur le nez, les lèvres et une bonne partie des joues. Parfois, la muqueuse buccale et celle des fosses nasales étaient également irritées. L'auteur crut d'abord qu'il s'agissait de brûlures par la chaux; mais la découverte d'un cas analogue chez un autre cheval, dont la face pelait et dont la bouche et les lèvres tuméfiées laissaient passer une salive abondante, épaisse et mousseuse, et chez qui la langue présentait de grandes lignes d'excoriation, comme à la suite d'une brûlure, amena l'auteur à inspecter la mangeoire de l'animal. Il y trouva de nombreux Blaps et dès lors il ne douta plus un instant que les lésions avaient été causées par les sécrétions de ces Ténébrionides, car, comme il le dit lui-même : "C'est à eux que j'avais songé devoir attribuer quelques grosses phlyctènes qui ont apparu deux ou trois fois sur la face d'un de mes enfants, sans autre cause appréciable. »

Le liquide âcre rejeté par des glandes situées au voisinage de l'anus du coléoptère aurait en effet la faculté de produire une vésication violente sur les tissus animaux les plus délicats.

Traitement. - Des gargarismes avec du chlorate de potasse furent prescrits pour guérir l'animal.

En Italie, Bassi (1893) a constaté les mêmes lésions sur des chevaux dont la litière était infestée par des Blaps mortisaga L. La présence de ceux-ci dans les écuries s'explique très bien du fait de l'écologie des Ténébrionides qui recherchent tout particulièrement les endroits obscurs.

Cet auteur fit des expériences qui confirmèrent le rôle irritant des coléoptères :

1. - Trois chevaux frottés à la lèvre supérieure avec l'abdomen d'un Blaps vivant montrèrent, au bout de 3 jours, sur la partie frottée, de petites vésicules et un plissement de l'épiderme noirci.

2. - Avec des Blaps morts, la même expérience se montra négative, le coléoptère mort perdant l'action irritante due au liquide huileux et âcre des glandes anales.

3. - Dans une écurie, de la paille infestée par des Blaps fut donnée comme litière à 1 cheval, 3 ânes et 1 vache.

Ceux-ci furent observés les jours suivant le début de l'expérience (27 juillet-4 août 1892). 
Chez le cheval, les symptômes furent les suivants :

29 juillet. - Hypersécrétion de la muqueuse nasale, léger épistaxis, légère inflammation de la glande intermaxillaire.

30 juillet. - Tuméfaction importante des lèvres et du pourtour des yeux, exfoliation épidermique du museau et présence de vésicules sur celui-ci; muqueuse nasale très rouge et couverte d'une exsudation jaunâtre.

31 juillet. - Légère amélioration.

$1^{\text {er }}$ aoùt. - Amélioration nette, mais muqueuse nasale toujours rouge (à partir de ce moment, le cheval fut changé d'écurie et il se rétablit rapidement).

L'auteur italien conclut que trois stades peuvent être observés dans l'évolution de ces lésions :

1. - Stade érythémateux. - Injection de la peau et de la muqueuse nasale.

2. - Stade vésiculeux. - Eruption de petites vésicules confluentes.

3. - Stade squameux. - Exfoliation épidermique plus ou moins étendue.

Il remarque aussi que ceci correspond aux lésions cutanées connues sous les noms de Herpes labialis, "Hitzausschlag », herpès phlycténoïde, rhinite pemphigoïde.

Ces propriétés irritantes de $B$. mortisaga ont été également mentionnées par Railliet (1895) qui rappelle qu'après l'exfoliation épidermique, il n'est pas rare de voir le pigment disparaitre sur certains points, laissant quelque temps des macules blanchâtres.

2. - Ane. - Bassi (loc. cit.) signale des lésions épidermiques dues à $B$. mortisaga, analogues aux précédentes.

3. - Vache.

a. - Endoparasite accidentel. - Roberts (loc. cit.) signale, à l'examen post-mortem, la présence de $\boldsymbol{O}$. granulatus précité, dans la panse de veaux du Queensland morts de 6 à 12 heures après les premiers symptòmes de maladie, sans pouvoir affirmer si le coléoptère était vraiment la cause du décès.

b. - Lésions externes. - Bassi (loc. cit.) a expérimenté l'action irritante de $B$. mortisaga sur une vache ; les mêmes symptòmes que chez le cheval et l'âne ont été observés, avec accentuation de là rhinite catarrhale et de la tuméfaction des naseaux (orifices du nez obturés). 
4. - Gamélidés.

a. - Endoparasite accidentel. - Blanchard (1898) a signalé la présence accidentelle de larves de Ergates faber L. (Cerambycidæ) dans les fosses nasales d'un dromadaire, au Yémen. Celles-ci ont probablement pénétré par erreur dans le mufle de l'animal, alors que celui-ci broutait des écorces sous lesquelles elles se trouvaient (1).

b. - Intoxications. - Indine et Frolow (1935) signalent l'intoxication de chameaux, en U.R.S.S., à la suite d'ingestion de Zonabris sp. (Meloidæ). Ce travail est malheureusement rédigé entièrement en russe, sans résumé accessible.

5. - Ghien. - Tran Van N'Ga (1938) (in Curasson, 1942) a signalé, au Tonkin, l'intoxication de chiens ayant mangé des oiseaux qui avaient ingéré des Cantharides (Meloidæx). On sait que les grenouilles et certains oiseaux sont en effet résistants à l'action de la cantharidine que contiennent les Méloïdes, mais qu'une fois ingérés par un carnivore, ils peuvent déterminer des accidents chez ce dernier.

Chez les chiens mentionnés par l'auteur indochinois, les symptômes observés étaient les suivants : vomissements, dysurie, urine sanguinolente et albumineuse émise fréquemment par petites quantités, excréments sanguinolents, muqueuses hyperhémiées, respiration et mouvements du cœur accélérés.

6. - Divers animaux domestiques. - Curasson (loc. cit.) signale qu'aux colonies, à la fin de la saison des pluies, des vols importants de Cantharides « peuvent empoisonner des mares, des abreuvoirs et causer des accidents graves de gastro-entérite hémorragique chez les animaux ».

Les symptômes sont les mêmes que ceux décrits ci-dessus chez le chien ; chez les ruminants, l'ingestion de Cantharides cause, en plus, « une salivation abondante, une inflammation douloureuse de la muqueuse buccale et de la dysphagie ».

Dans l'Inde, les propriétés toxiques des Cantharides sont utilisées criminellement pour empoisonner le bétail : on mêle de la poudre de ces coléoptères aux aliments des animaux (Curasson, loc. cit.).

(1) Mon ami P. de Miré me signale qu'au Sahara occidental un Bostrychide indéterminé (nom indigène : " sousse ») causerait des abcès de l'oreille externe des dromadaires entrainant généralement la mort. Cette maladie est si répandue qu'à l'époque de l'éclosion de ces coléoptères (juillet-août) les indigènes évitent de faire brouter aux Camélidés Leptadenia pyrotechnica (Asclépiadacée) d'où les insectes semblent provenir. 


\section{Oiseaux}

\section{1. - Poules et poulets.}

a. - Lésions externes. - Mégnin (1901) rapporte un cas insolite d'infestation d'un poulailler, aux environs de Toulouse, par des Tenebrio moliton L. (Tenebrionidx), qui s'attaquaient aux pattes des poules couveuses ; les lésions causées par le mordillement répété de ces coléoptères étaient telles que l'éleveur trouva «les œufs tout couverts de sang, et souvent les poules exsangues et mortes ».

L'auteur conclut pour expliquer ce cas : "Non seulement les vers de farine adultes dévorent des substances végétales amylacées - ce que tout le monde sait - mais ils s'attaquent aussi aux substances mortes et aussi aux oiseaux vivants qu'une fonction spéciale, l'incubation, a immobilisés. 》

b. - Intoxications. - Aux U.S.A., Macrodactylus subspinosus Fab. (Scarabridæ) est chaque année la cause de la perte d'un grand nombre de poulets. Lamson (1916) signale qu'en 1909 plusieurs centaines d'entre eux périrent ainsi ; des expériences furent alors entreprises dans une Station expérimentale. L'on croyait tout d'abord que la mort de ces oiseaux était due à une cause mécanique (perforation ou morsure du jabot par contact avec les pattes ou les pièces buccales du coléoptère). Mais l'on réalisa bien vite que les volatiles mouraient intoxiqués, ce qui fut confirmé par les expériences suivantes :

1. - Un filtrat d'extrait de ces insectes écrasés et d'eau distillée fut donné aux poulets par voie buccale, à l'aide d'un compte-gouttes, et de nombreuses morts en résultèrent, surtout parmi les plus jeunes individus. L'examen post-mortem ne montra pas d'anormalité des organes. Les individus plus âgés vécurent, mais présentèrent des symptômes d'empoisonnément.

2. - La toxicité des coléoptères fut confirmée par l'expérience suivante :

Un extrait fut préparé de $40 \mathrm{gr}$. de coléoptères pour $60 \mathrm{cc}$. d'une solution saline à $0,9 \%$; celui-ci fut centrifugé 5 minutes, puis aspiré dans une pipette et filtré sous vide. 3 cc. de cet extrait injectés en intraveineuse à un lapin de $690 \mathrm{gr}$. déterminèrent sa mort en 6 minutes. D'autres injections à des lapins, à différentes doses, eurent le même effet, et l'auteur conclut que le poison doit être une neurotoxine agissant sur le cœur des poulets et des lapins.

Gallagher (1920) a précisé ces données et, reprenant les observations de Lamson, il indique les doses léthales de coléoptères ingérés, suivant l'àge des volatiles. 
Les poulets âgés de plus de $\mathbf{1 0}$ mois ne sont pas affectés par l'ingestion des insectes, mais 15 à 20 de ceux-ci suffisent pour tuer un poulet d'une semaine, et 96 pour un de 10 semaines.

L'auteur américain décrit les symptômes observés qui peuvent apparaitre dès une heure après l'ingestion des Macrodactylus : l'oiseau devient alourdi, faiblit sur ses pattes et tombe sur le côté ; il peut y avoir des convulsions avec cris aigus poussés par l'animal et rétraction caractéristique de la tête et du cou sur le dos. La mort peut survenir de $1 / 2$ heure à 24 heures après l'observation des symptômes. Si les poulets n'ont pas absorbé une quantité suffisante d'insectes pour produire la mort, les symptòmes disparaissent.

A l'autopsie, aucune modification du tube digestif et des autres viscères ne fut observée; seuls, les vaisseaux sanguins de la paroi du cœur se montrèrent injectés.

Traitement. - L'auteur signale que l'action rapide de la toxine rend la guérison difficile chez des poulets présentant déjà des symptòmes d'intoxication. Un purgatif à base d'huile de ricin et l'emploi de sels d'epsom sont recommandés. Comme moyen préventif, il est recommandé d'éloigner les poulets des endroits où peuvent se rencontrer les coléoptères et de tuer ces derniers à l'aide d'arséniate de plomb et de mélange de Bordeaux.

2. - Pigeons. - Neveu-Lemaire (1912 et 1938) signale que des larves de Necrophorus vestigator Her., Silpha (Oeceoptoma) thoracica Gang. (Silphidæ) et Dermestes lardarius L. (Dermestidæ) (1), espèces normalement saprophages ou nécrophages, peuvent se développer sur le fumier des colombiers et attaquer des pigeonneaux dont elles « rongent la peau du cou et du ventre et même les muscles sous-jacents. Les plaies ainsi produites sont envahies secondairement par des larves de mouches et deviennent souvent mortelles ».

Traitement. - " On enlève les larves de la surface des plaies, en appliquant sur celles-ci un pansement antiseptique à l'eau phéniquée ou lysolée au dixième. 》

Ainsi donc, ces quelques cas montrent que les coléoptères présentent un certain intérêt en médecine vétérinaire.

Pour terminer, nous donnerons sous forme de tableau la récapitulation des principaux cas signalés $(\mathrm{L}=$ larve ; $\mathrm{A}=$ adulte) :

(1) Ce parasitisme accidentel des larves et adultes de $D$. lardarius ainsi que de ceux de $D$. bicolor $F$. chez les jeunes pigeons est également mentionné par von Lengerken (1924) (in Schulze : Biol. Tier. Det:/sch. Coleoptera, 40, 10, p. 24) qui rappelle aussi l'attaque accidentelle des poules couveuses par Tenebrio molitor L. 


\begin{tabular}{|c|c|c|c|c|c|c|c|c|c|c|c|}
\hline 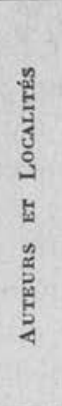 & 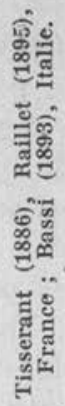 & 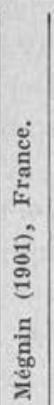 & 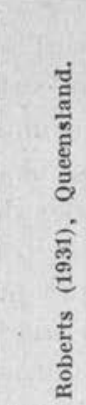 & 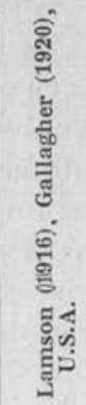 & 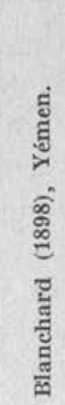 & 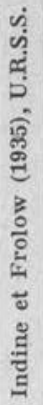 & 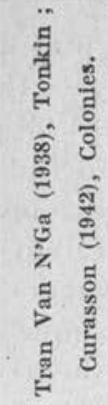 & & 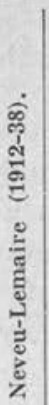 & 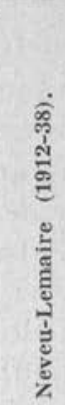 & 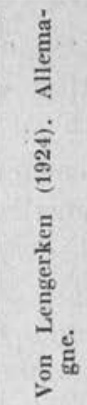 \\
\hline 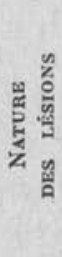 & 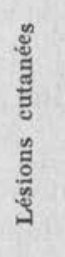 & 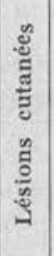 & 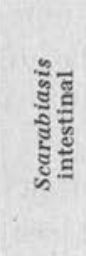 & हूँ & 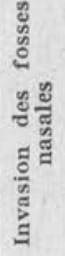 & 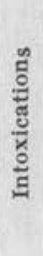 & 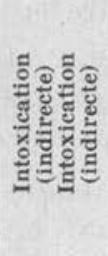 & & 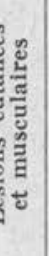 & 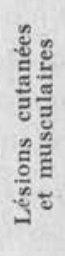 & 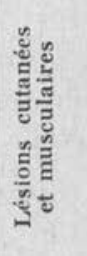 \\
\hline 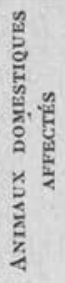 & 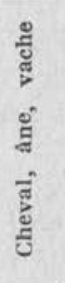 & 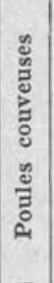 & 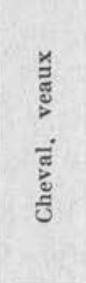 & 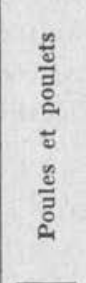 & 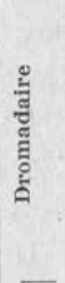 & है & ⿷匚 & 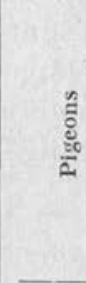 & 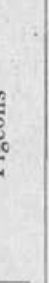 & $\begin{array}{l}\text { हैं } \\
\frac{0}{2}\end{array}$ & $\frac{\tilde{B}}{a}$ \\
\hline है & 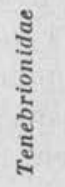 & 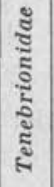 & 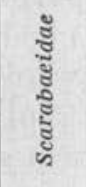 & 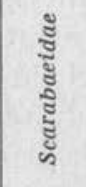 & 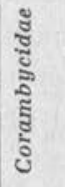 & 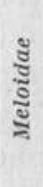 & 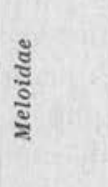 & : & 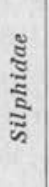 & 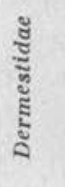 & 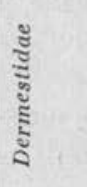 \\
\hline 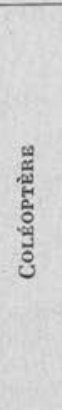 & 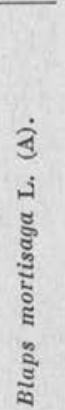 & 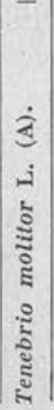 & 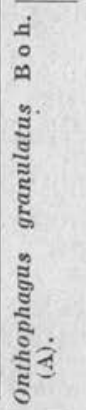 & 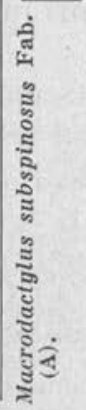 & 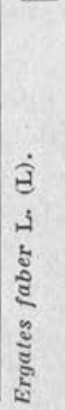 & $\begin{array}{l}\dot{3} \\
\dot{1} \\
\frac{2}{2} \\
\frac{3}{3} \\
\text { हू. }\end{array}$ & 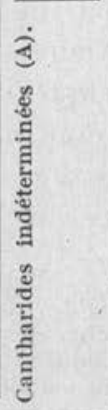 & 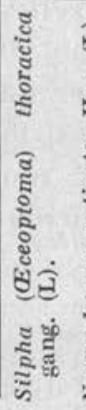 & 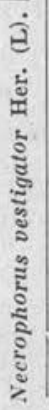 & 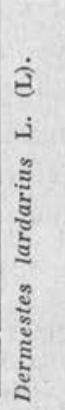 & 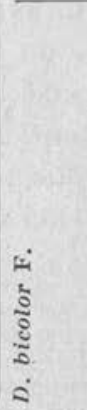 \\
\hline
\end{tabular}




\section{BiBLIOGRAPHIE}

1893. - Bassi (R.). - Eczema vescicoloso labiale e nasale del cavallo causato in maniera indiretta dal Blaps nero (B. mortisaga L.). $1 l$ Mod. Kooiat., 1893, 63-68.

1890. - Blanchard (R.). - Traité de Zoologie Médicale. 2 vol., Baillière édit., Paris.

1898. - Blanchard (R.). - Sur des larves de coléoptère longicorne trouvées dans les fosses nasales d'un dromadaire. Arch. Parasitol., I, 513-14.

1942. - Curasson (G.). - Traité de Pathologie Exotique, Vétérinaire et Comparée. 3 vol., Vigot édit., Paris.

1920. - Galiagher (B. A.). - Rose-chafer poisoning in chickens. J. Amer. Vet. Assoc., LVII, 692-95,

1935. - INDine (S. C.) et Frolow (A.). - L'intoxication des chameaux par les Méloïdes (en russe, sans résumé). Sovietsk. Veter., 1935, 38-39.

1916. - Lamson (G. H.). - The poisonous effects of the rose-chafer upon chickens. Science, n.s., XLIII, 138.

1901. - MÉgxin (M.). - Un cas extraordinaire de parasitisme du Tenebrio molitor. C.R. Soc. Biol., LIII, 834.

1912. - Neveu-Lemaire (M.). - Parasitologie des animaux domestiques, Lamarre, Paris.

1938. - Neveu-Lemaine (M.). - Traité d'Entomologie Médicale et Vétérinaire. Vigot, Paris.

1895. - Ranliet (A.). - Traité de Zoologie Médicale et Agricole. Asselin et Houzeau, Paris.

1931. - Ronents (F. H. S.), - The occurrence of a beetle (Onthophagus granulatus Boh.) in the stomach of domesticated animals. Queensl. Agric. J., $\mathrm{XXXV}, 171$.

1948. - THḰodonidès (J.). - Les coléoptères parasites accidentels de l'homme. Ann. Parasit., XXIII, 348-63.

1886. - Tisserant (H.). - Accidents provoqués chez le cheval par le Blaps mortisaga. J. Méd, Vèt. Kootechn., 1886, 25-32.

1938. - Tran VAN N'GA. - Intoxication indirecte par des cantharides due à la consommation de viande d'oiseau. Cahiers Vét. Tonkin, déc. 1938. (Cette référence n'a pu être consultée).

Muséum National d'Histoire Naturelle de Paris.

\section{Errata graviora}

Tome XXIII, 1948, Mémoire ThÉonoridès, p. 356, ligne 13, lire : Acinopus picipes, au lieu de A. picipesœ; p. 357, lignes $5,25,31,34$ et p. 361 , dernière ligne du résumé, lire : Niplus hololeucus, au lieu de $N$. holoceucus, 